\title{
Modular Multilevel Converter Controlled by Daisy- Chain Model Predictive Control for Medium Voltage Shunt Active Filter
}

\author{
Mohamed A. ElMansy, Mostafa S. Hamad and Alaa Eldin A. Khalil \\ Arab Academy for Science, Technology and Maritime Transport, Alexandria, Egypt
}

\begin{abstract}
Nowadays most of the loads exhibits a non-linear nature leading to sever distortion in the grid current. Mitigation of the adverse effects of such problems has been the focus of numerous researches throughout the past century. However, each genuine concept has its set-backs regarding energy consumption, reliability or protection. A Modular Multi-level converter (MMC) based Shunt Active Power Filter (SAPF) is introduced in this paper. The converter is to be connected to the medium voltage grid to compensate the reactive power required by the non-linear loads, thus eliminating the harmonics. The MMC out performs high-voltage/highpower converters in terms of quality output performance, high modularity, simple scalability, and low voltage and current rating demand for the power switches. A Model Predictive Control (MPC) algorithm is developed to control the converter. Least Mean Square Algorithm (LMS) based Adaptive Linear Neuron (ADALINE) network generates the reference current. The threephase load current is measured and analyzed to estimate the harmonics waveform, which is then fed to the MPC to generate the optimal switching sequence for the MMC. Simulation and results are presented to show the effectiveness of the proposed techniques.
\end{abstract}

Keywords: Power quality; Modular Multilevel Converter (MMC); Shunt Active Power Filter (SPAF); A Model Predictive Control (MPC); Adaptive Linear Neuron (ADALINE)

\section{Introduction}

Switching devices form the basis of most of the devices used in the industry or pedestrian use. Rectifiers, inverters, motor drives and battery chargers are all forms of non-linear loads which typically induce current harmonics.

Eq.(1) shows the total current signal with harmonics. There are three types of harmonics sequences; Positive sequences as $\left(1^{\text {st }}, 4^{\text {th }}, 7^{\text {th }}, \ldots\right)$ has an orientation direction which is same as the fundamental signal, negative sequence harmonics $\left(2^{\text {nd }}, 5^{\text {th }}, 6^{\text {th }}, \ldots\right)$ their orientation is opposite to the fundamental signal. Triplens have zero-sequence; they circulate between source and neutral.

$$
I_{\text {Total }}=I m_{h} \cdot \sin \sin (w t)+I m_{3} \cdot \sin (3(w t+\delta)+\cdots
$$

The reactive current component drowns from the bus bar leads to convoluted complications. First the power quality is severely reduced given the wasted power. Positive sequence harmonics causes overheating of devices, wires and conductors, while negative sequence distorts the magnetic field, affecting the generation side (Ortmeyer et al., 1985). Various techniques have been developed for Harmonic elimination, they can be classified as passive filtering, active filtering or hybrid filtering. Passive filters are networks of resistors, capacitors, and inductors, where the nature of such elements is exploited to block a specific band of 
frequencies (Haur et al., 2020). Different topologies of traditional low pass filters are shown in Figure 1

Figure 1:Low Pass Passive Filters

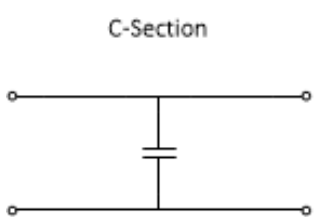

RC-Section

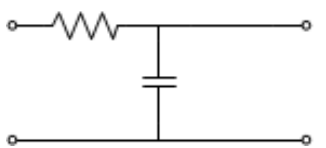

L-Section

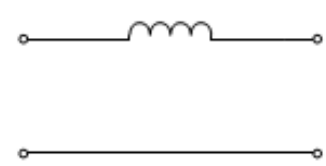

CR-Section

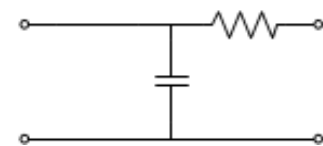

LC-Section

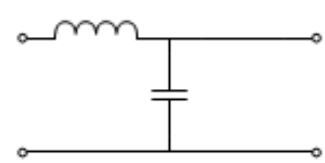

Pi-Section

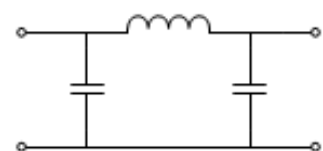

CL-Section

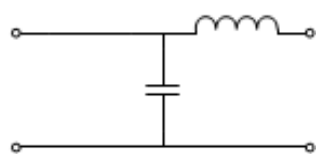

T-Section

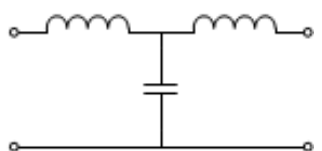

Their complexity ranges from simple first order filter to $3^{\text {rd }}$ order filters, which offers better filtering efficiency. Higher order can be generated either by using topologies such (e.g., Pisection, T-section) or by combining two or more of the lower order filters. The prevailing type of filter used is the second order LC-filter where its cut-off frequency can be calculated using Eq.(2)

$$
F_{c u t t-o f f}=\frac{1}{2 \pi \sqrt{L C}}
$$

Although the passive filters enhance the power quality, they are easy to design and relatively cheap. Passive filters are bulky and provide fixed compensation with generating resonance problems. So, if the harmonic requirement changes with load, passive filters will not be able to handle it but will need to be re-tuned. Shunt Active Power filters (SAPF) are another remarkable technique used to eliminate harmonics.

SAPF consists of VSI which converts a DC of a capacitor, battery bank or PV system to AC and injects it to the PCC to compensate the harmonics required by the non-linear loads. Thus, SAPF that it doesn't need to be tuned at a specific frequency as done with a passive filter which ensures its high reliability. Thus, the dynamic behavior of the SAPFs out-performs that of the passive filters with reliable control SAPF can be controlled to pass a specific band while attenuating the rest (Kalaignan \& Raja, 2010).

Added to that, it can handle voltage sags and swells and compensate reactive currents. One final advantage is that resonances will not take place with the facility distribution systems. Active filtering could be comparatively new technology. There's still a need for supplementary analysis and research to make this technology well proven (Jacob et al., 2014).

Two influential factors need to be considered while designing any SAPF. First, the injected current harmonics depends on the capacitor's voltage of the inverter, as a result, the capacitor's voltage should be higher than the PCC voltage. Typically, it reaches twice the PCC voltage. Second, to maintain the stability of the whole system, the capacitor's voltage should contain fewer ripples. The higher capacitor ratings the better for the stability and fewer ripples for capacitor's voltage, however this means bigger size, higher cost, and less reliability of the capacitor. 


\section{$4^{\text {th }}$ International Conference on Modern Research in Science, Engineering and Technology}

\section{5-7 MARCH 2021 \\ BERLIN, GERMANY}

Figure 2: Shunt Active Power Filters General Topology

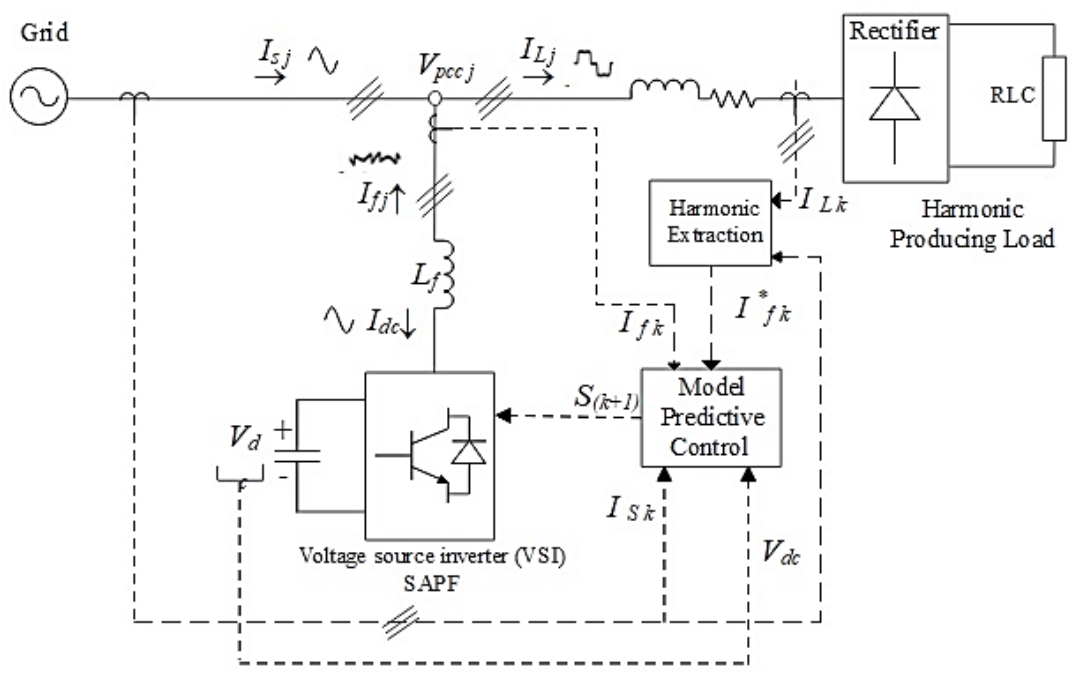

As mentioned previously MMC exhibits and outstanding performance compare to other high-voltage/high-power converters. Thus, a MMC based SAPF would be an insensible tool to eliminate harmonics in generation systems. A PI controller was designed and tested in (Adam et al., 2010) (Madi et al., 2018). In this paper, a MPC controller for monitoring the grid conditions and control the MMC. MPC enables the MMC based SAPF to work on a wide range of loads without being affected by any inductive or capacitive changes in Medium Voltage application.

MMC theory of operation and applications are discussed in (Chuco \& Watanabe, 2011)(Vazquez et al., 2014). The superiority of the MMC over the conventional VSC manifests in overcoming high rate of change of converter arms current, over-voltages on semiconductors components, electromagnetic radiation, and power loss in PWM of semiconductors (Del Moral, 2020). MMC modularity offers sub-module redundancy and flexibility in specifying voltage level and controlling the injection method for getting optimum results, specifically for HV/MV applications. It is worthy to mention that the switching frequency is changeable with the number of SM which can be controlled to maintain a good performance with the least power loss. Arm inductance is a major contributor in limiting the DC short circuit current (Coordination et al., 2012), its selection is discussed in (Zygmanowski et al., 2013).

MPC is a compact controller system capable of controlling multiple variables, thus a much simpler system hardware configuration. In this study, MPC is used to control the three critical points of MMC: SM voltage stability, MMC circulating current and AC output signal. Several techniques were studied to control the MMC via and MPC algorithm (Perez et al., 2012) (Moon et al., 2015). In this paper, MPC is a one-stage controller without PWM or carrier signal. In addition, the usage of a PI controller to maintain converter capacitor voltage is avoided. Also, new technique will be shown regarding filtering an estimated signal instead of using sensors thus drastically reducing the cost.

SAPF always requires an accurate reference signal to get the best results. Adaptive Linear Neuron (ADALINE) which discusses neural nets for adaptive filtering and adaptive pattern recognition is used for many applications as digital communications and biomedical systems, it also plays a significant role in digital signal processing (DSP). Many types of research had been carried out discussing the concept of ADALINE. In this application, a double algorithmic ADALINE will be used for harmonics extraction and processing, from the MV application for 
guaranteeing robust reference for MPC. Many papers discussed the idea of ADALINE (Widrow \& Winter, 1988)(Cherif et al., 2019), but few papers talked about using ADALINE as a noise reference for MPC converter in SAPF application. Each of ADALINE, MMC, and MPC influences Harmonic elimination performance and cost which will be shown in the next sections.

\section{System Design}

\subsection{Modular Multilevel Converter}

Modular Multilevel Converter (MMC) has proven to be among the most suitable converters for medium/high power applications. Its modularized structure enables it to conquer short comings that manifest in other topologies. First, MMC generated less harmonics compared to the conventional (2-Level) converter. Additionally, owing to the multiple sub-modules structure the equivalent frequency can be maintained high with reduced switching losses compared to the conventional (2-Level) converter particularly in medium and high-power applications. All things considered, as the number of submodules increased the lower the switching losses are and better the performance. Further elucidation on how MMC surmounts the drawbacks of the 2-Level conventional converter is discussed in (Perez et al., 2012).

High frequency trades of the efficiency and the EMI for a reduced the size of passive elements and a faster and more robust transient response. The switching power loss increases with higher switching frequency due to the high number of turn-on and offs. Also, EMI is a major problem as the EMI from a switch is proportional to the square of the switching frequency. In conclusion, high switching frequency can affect negatively the efficiency of any converter.

In practice, the equivalent switching frequency of MMC can be calculated via Eq.(3), where $f_{s w}$ is the switching frequency of a submodule.

$$
f_{s w-e q}=n f_{s w}
$$

The mentioned advantages of MMC show that SAPF is one of the best applications to use MMC. It is reliable to divide the output signal into several voltage levels which are reflected in the number of SM. The higher the number of SM used, the less switching frequency, which maintains high converter efficiency, compared to the conventional converter, and lowers harmonic content in the output signal.

SAPF as an application for harmonic elimination requires a low harmonic injected signal. As discussed in reference (Martinez-Rodrigo et al., 2017), it is possible to divide the output signal based on voltage level. One of several SM is that the more SMs exist, the more voltage levels (Staircase) the output signal can be configured which increases the precision of the output signal and decrease the EMI effect.

In this article, 5-level MMC shown in Figure 3 will be considered as an application and case study. The output voltage of each of the 5 sub-modules is used as a control reference for specifying both SM voltage balancing and output desired current.

In this research, the algorithm is self-configured based on the number of attached SM for additional reliability hardware. One of the control problems of MPC is that the multi-variable control at cost function acts as a trade-off between the controlled variables, as a result, at some operational conditions, it is not the best idea to have a cost function with multiple-variable especially if these variables are affecting the desired signal. 
To solve this problem, this research is based on the "Modular Daisy Chain" algorithm control for MPC. This technique maintains an independent control approach for each variable, included in the same controller. The used technique highlights the reliability of algorithmic control.

Figure 3: 5-level Modular Multilevel Converter MATLAB Simulink Model

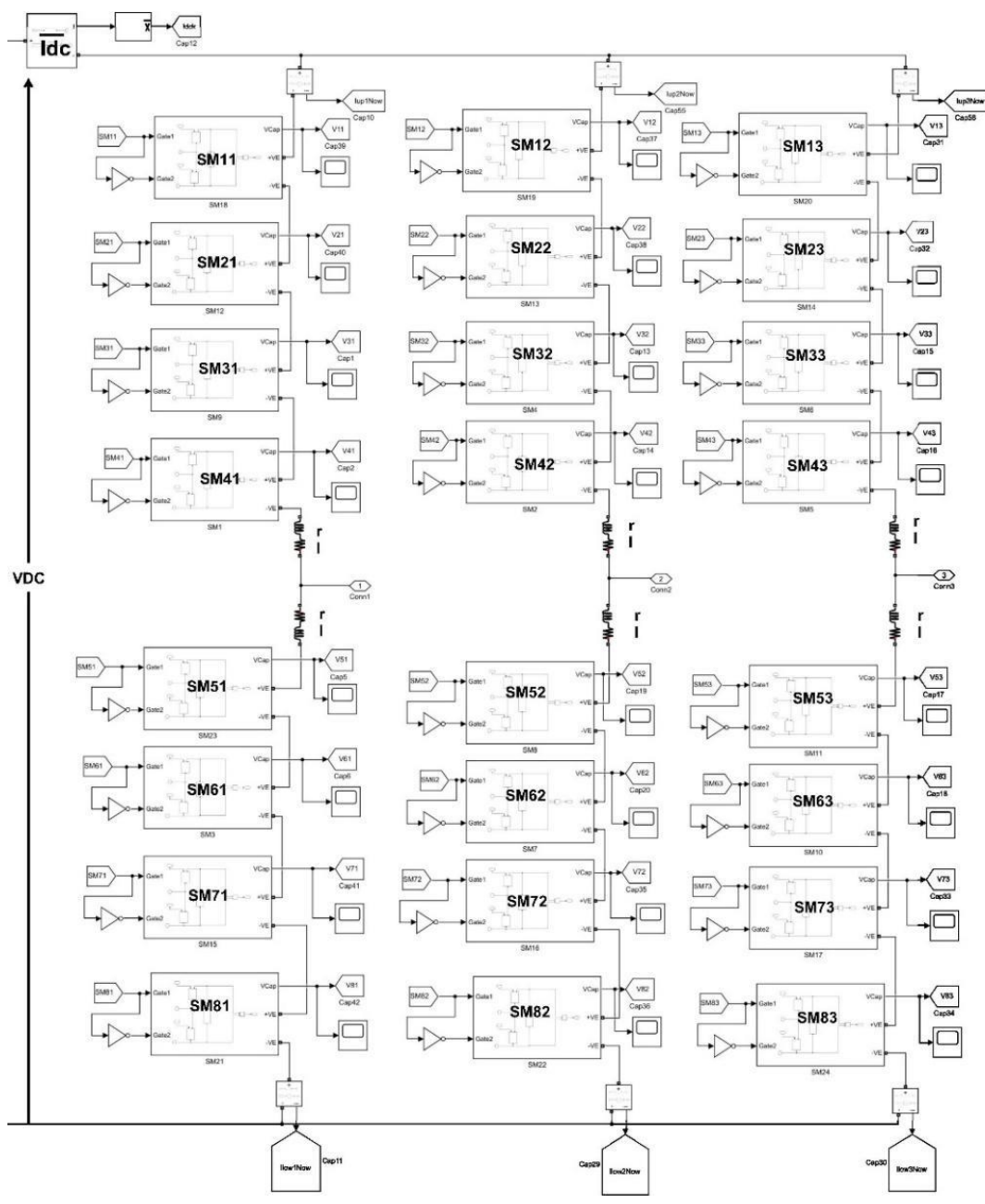

\subsection{Modeling of MMC for MPC using "Modular daisy chain" Algorithm}

The base of the system model is Applying Kirchhoff's Voltage Law (KVL) between the bus bar up to the point of common coupling with the grid, while considering the circulating current $I_{\text {circ }}$ which can be calculated through Eq. $(4$.

$$
\begin{gathered}
I_{u p, k}=-\frac{I_{f, k}}{2}+I_{\text {circ }, k} \\
I_{\text {low }, k}=\frac{I_{f, k}}{2}+I_{\text {circ }, k}
\end{gathered}
$$

$k$ represents the phase (e.g.; a, b or c), $I_{f}$ is the output MMC current, $I_{\text {up }}$ and $I_{\text {low }}$ are the currents flowing in the upper/lower arms of MMC for each phase. So that $I_{u p, a}$ is the current passing in the upper arm of the first phase, and $I_{l o w, a}$ is the current passing in the lower arm of the first phase.

The existence of the MMC circulating current is due to the fluctuation of the capacitor voltage of each SM. Additional analysis of circulating current is studied in references (Rohner 
et al., 2010)(Hoon et al., 2019)(Tu et al., 2011). Applying KVL and substituting Eq.(4), the following results:

$$
V_{p n, k}=V_{m m c, k}-\left(R * I_{f, k}\right)-L \frac{d I_{f, k}}{d t}-\left(\frac{r}{2} * I_{f, k}\right)-\left(\frac{l}{2} * \frac{d I_{f, k}}{d t}\right)
$$

Using Euler approximation and discretizing the predicted MMC output current can be formulated as follows, where $T_{s}$ is the sampling time.

$$
I_{f, k}\left(t+T_{s}\right)=\frac{1}{R+\frac{r}{2}}\left(V_{m m c, k}\left(t+T_{s}\right)-V_{p n, k}\left(t+T_{s}\right)+\left(\frac{\left(L+\frac{l}{2}\right)}{T s} I_{f, k}(t)\right)\right)
$$

In this application, four sub-modules are used in each arm, as a result, However, generally speaking the MMC generated voltage is divided into several levels according to the number of Sub-modules used:

$$
V_{m m c, k}=\frac{V_{d c}}{N} * X
$$

$X$ is the index voltage level, $N=4$.

$$
\begin{gathered}
V_{u p, k}{ }^{*}=\frac{V_{d c}}{2}-V_{m m c, k}{ }^{*} \\
V_{l o w, k}{ }^{*}=\frac{V_{d c}}{2}+V_{m m c, k}{ }^{*}
\end{gathered}
$$

As a result, the cost function of the main output current:

$$
J_{\text {current }}=\left|I_{f, k}{ }^{*}\left(t+T_{s}\right)-I_{f, k}\left(t+T_{s}\right)\right|
$$

Eq.(8) will be used to contribute finding the least level of unbalanced current $I_{\text {diff }}$ as mentioned in (Coordination et al., 2012).

$$
\begin{aligned}
& I_{\text {diff }, k}\left(t+T_{s}\right)=\left(\frac{2}{R+\frac{L}{T_{S}}}\right)\left(V_{d c}+\left(\frac{l}{T s} I_{d i f f, k}(t)\right)\right) \\
& -\left(V_{\text {low }, k}{ }^{*}+2 V_{\text {diff }, k}\left(t+T_{s}\right)+V_{u p, k}{ }^{*}\right) \\
& V_{\text {diff }, k}=\frac{V_{d c}}{N} *\left[\begin{array}{lll}
-1 & 0 & 1
\end{array}\right]
\end{aligned}
$$

Eq.(11) is based on 3 levels calculation to reach the least level of unbalanced current. The cost function for the least unbalanced current/circulating current:

$$
J_{\text {circ }}=\left|\frac{I_{d c}{ }^{*}\left(t+T_{s}\right)}{3}-I_{\text {diff }, k}\left(t+T_{s}\right)\right|
$$

The optimum values of $V_{\text {diff }}$ will be taken into consideration for voltage reference of the upper and lower arm of MMC. The next step is choosing the optimum sub-modules to be in service, depending on their level of charge. Switched ON sub-modules can be expressed as:

$$
V_{d c, k}\left(t+T_{s}\right)=V_{d c}(t)+\left(i_{m}(t) * \frac{T_{s}}{C}\right)
$$




$$
J_{s m}=\frac{I_{a r m}}{C} * T_{s} *\left(\frac{V_{d c}}{N}-V_{d c, k}\left(t+T_{s}\right)\right)+S W *(\Delta S)
$$

Where $I_{\text {arm }}$ is the current passing at the upper or lower arm for SM selection, $S W$ is the adaptive weight that changes and is set to 0 as the constant switching frequency. A lot of research studied Model Predictive Control (MPC) with different algorithmic techniques reaching the desired performance (Oleschuk et al., 2002). In addition to, MPC with SAPF as (Massoud et al., 2004). The Flow chart in Figure 4 represents the flow of the MPC control algorithm based on the aforementioned discussion.

Figure 4: Flow Chart of MPC Algorithm
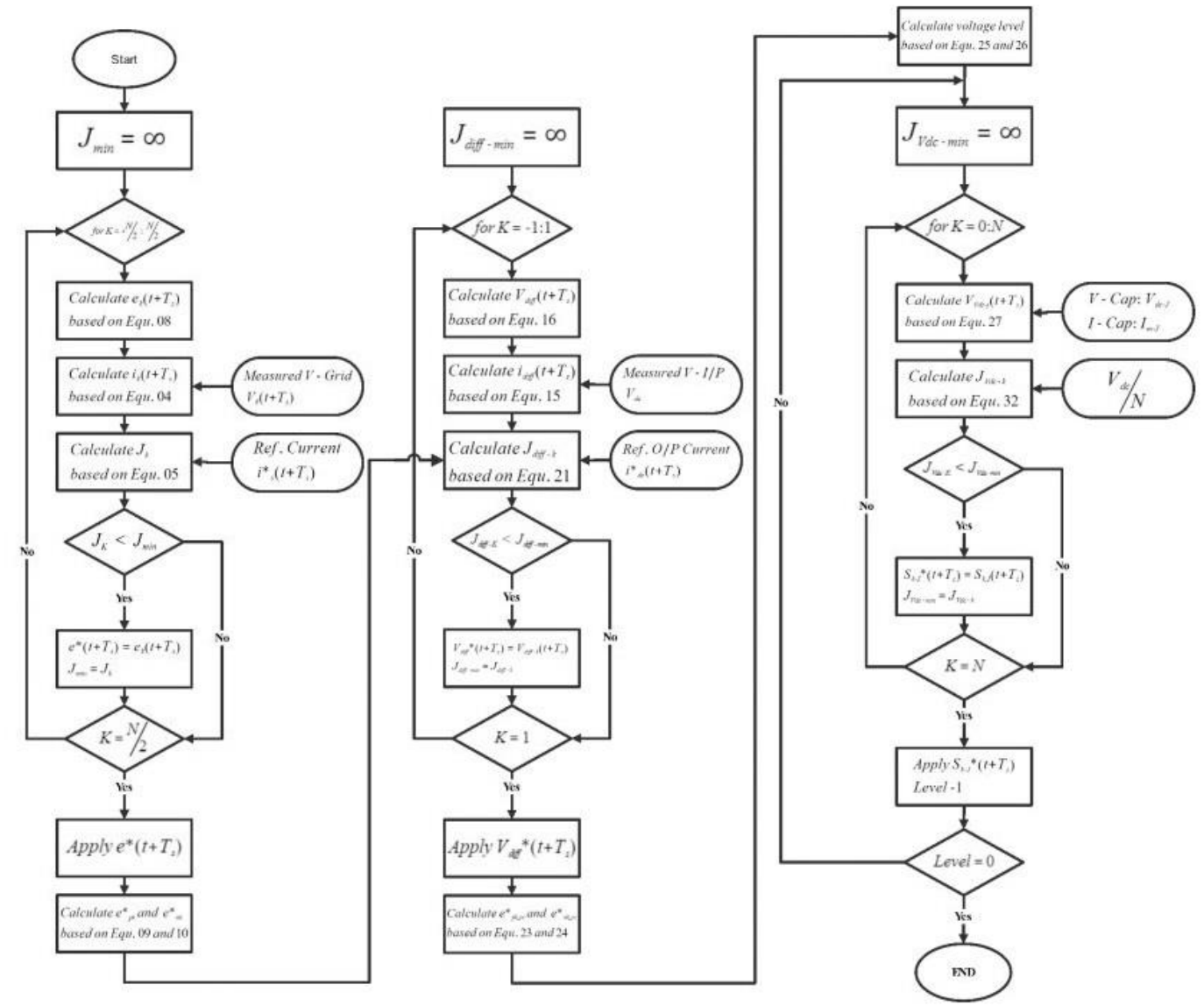

\subsection{ADALINE Least Mean Square (LMS)}

In order to generate the required the required reference current a double ADALINE is used. Using MPC with SAPF will substituting the conventional controllers of SAPF as PI and PWM. In addition, a PI controller for voltage balancing of MMC is not needed.

At first, it's vital to say the ennobling idea of this technique that came from the concepts of the organic model of living organic neurons. ADALINE theory of operation in addition to electrical applications are mentioned and discussed in (Widrow \& Winter, 1988)(Cherif et al., 2019).

Figure 5 shows the control loop of ADALINE LMS. Minimizing the "mean square error" means minimizing the square of the error so positive and negative error are treated equally and 
the main objective is "Zero error". In our application, "Least Mean Square" will be used. In this research, ADALINE is used for different purposes.

Figure 5: ADALINE LMS

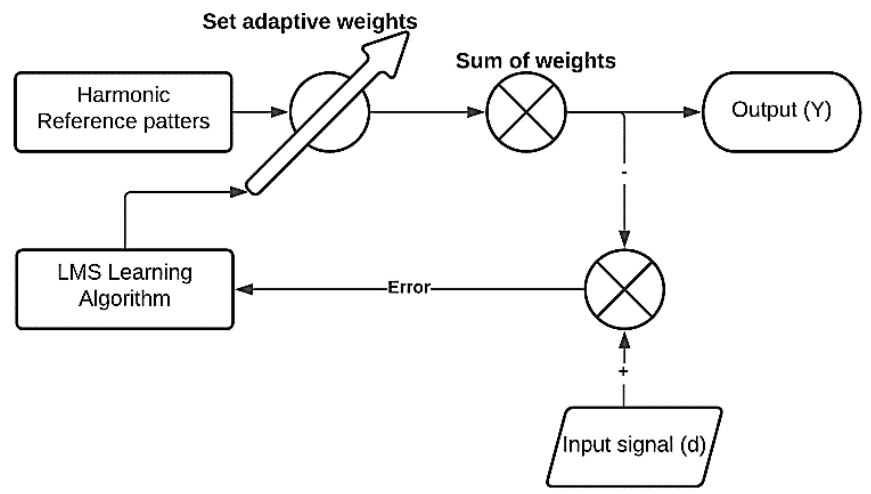

\subsection{ADALINE for extracting the needed Harmonics:}

The load current is used as an input for ADALINE in order to have both noise and fundamental signal as outputs. ADALINE mainly consists of 5 main variables:

$>$ Input Reference Pattern (X).

$>$ Adaptive weights (W).

$>$ Output (Y).

$>$ Learning Factor $(\eta)$

$>$ Input signal $(\mathrm{d})$

The Input Reference Pattern can be represented as follows;

$$
X=\sin \left(h_{i} \omega\right) \cos \left(h_{i} \omega\right)
$$

Where $h_{i}$ is the harmonic order $h_{i}=\left[\begin{array}{llll}1 & 3 & 5 & \cdots\end{array}\right]$

Initializing the weight factor (W), it is set as a small number (0.001) or less for every reference harmonic $h_{i}$. Setting the initial value of weight for each harmonic order is dependent on the application and the desired harmonic to be cured. In this research, all harmonics are treated equally for signal elimination. In some applications, it is important to specify a higher weighting factor for a specific harmonic order. The output is represented as a mathematical relation between the weight and harmonic reference.

$$
Y=\sum_{i}^{N}\left(\mathrm{~W} X^{T}\right)
$$

Where $\mathrm{N}$ is the maximum desired harmonic order.

Figure 6: Flow Chart ADALINE Algorithm 

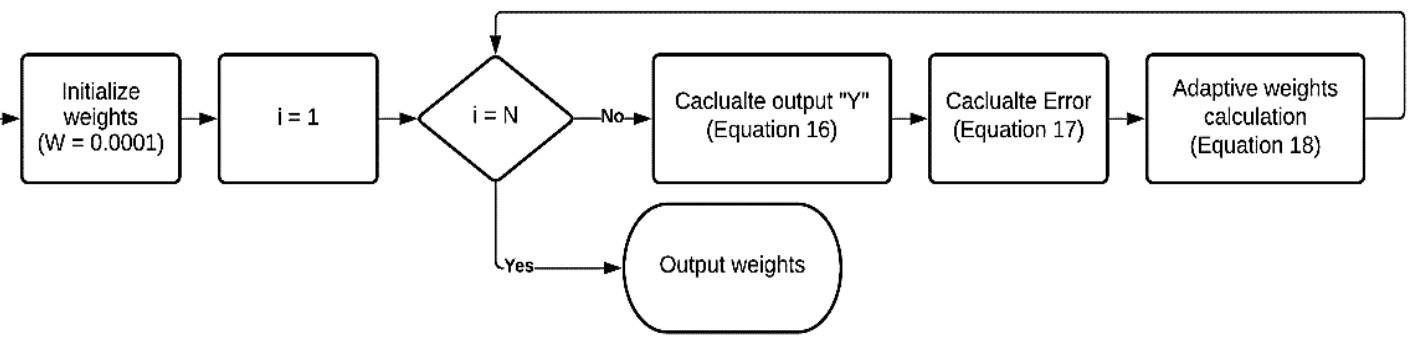

The next step is to calculate the error between the input signal represented as the load current and the output signal:

$$
\begin{gathered}
\varepsilon=d-Y \\
w_{j+1}=w_{j}+2 \mu \varepsilon_{j} X_{j}
\end{gathered}
$$

Eq.(18) represents the adaptive equation with a learning factor $\mu$ which guarantees the fastest decay of error. The learning factor is adjusted with the number of iterations for reaching the best result. In the ADALINE algorithm, the mean square error (MSE) between the reference signal and the load current is meant to decrease to its least level in order to reach the best reference signal for MPC.

In the SAPF application we will use a double algorithm of ADALINE for a good reference signal without phase shift to be MPC reference. This technique maintains a robust reference over wide power range, unlike using PI or MPC controllers which proved to be a good controller with a limited power range. PI controllers need tuning with changing the power application, while MPC is useless without that tuning. However, double ADALINE allowed independency on the power of the load. Figure 6 illustrates the algorithmic of each ADALINE showing the followed steps during the study of the case study. Figure 7 represents schematic double ADALINE input-output. Double Adaline inputs are Load current and network phase angle extracted by Phase-Locked Loop (PLL) or any phase angle hunting techniques.

Figure 7: Double ADALINE

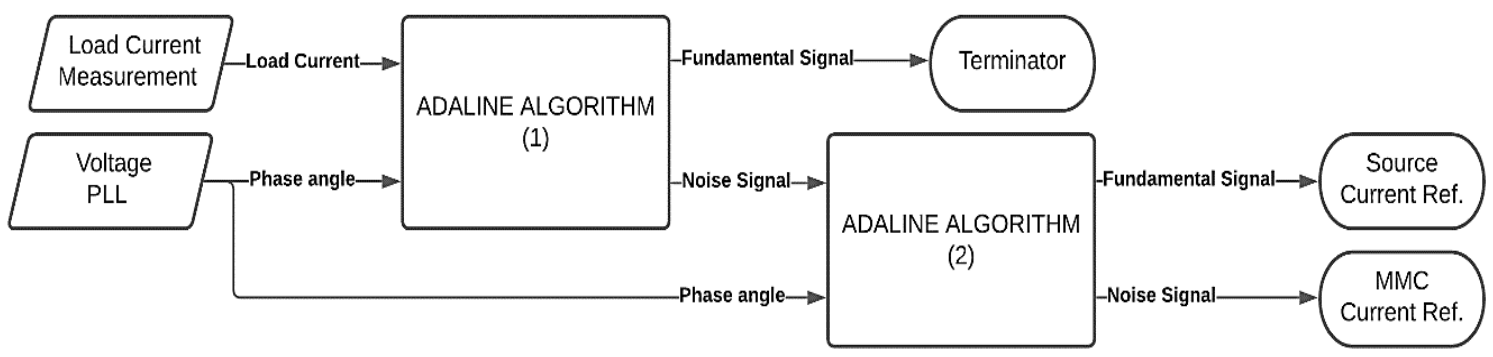

\section{Simulation and Results}

\subsection{System Parameters}

The research is done on a Medium voltage application. Figure 3 shows the used 5 Level $\mathrm{MMC}$ and

Figure 8 shows the whole system represented on MATLAB. Table 1 represents the values with which the design used for MMC.

Table 1: Parameters Table

\begin{tabular}{|c|c|c|}
\hline Parameters & Symbols & Value \\
\hline Number of submodules & $\mathrm{N}$ & 4 \\
\hline
\end{tabular}


$4^{\text {th }}$ International Conference on Modern Research in Science, Engineering and Technology

5-7 MARCH 2021

BERLIN, GERMANY

\begin{tabular}{|c|c|c|}
\hline The voltage of the converter at the DC side & Vdc & $5000 \mathrm{~V}$ \\
\hline Arm Resistance & $\mathrm{R}$ & $0.01 \Omega$ \\
\hline Arm inductance & $\mathrm{L}$ & $1.33 \mathrm{mH}$ \\
\hline Coupling Resistance & $\mathrm{R}$ & $0.044 \Omega$ \\
\hline Coupling Inductance & $\mathrm{L}$ & $9 \mathrm{mH}$ \\
\hline SM Capacitance & $\mathrm{C}$ & $4 \mathrm{mF}$ \\
\hline Fundamental Frequency & $\mathrm{f}$ & $50 \mathrm{~Hz}$ \\
\hline
\end{tabular}

Figure 8: SAPF system using MATLAB

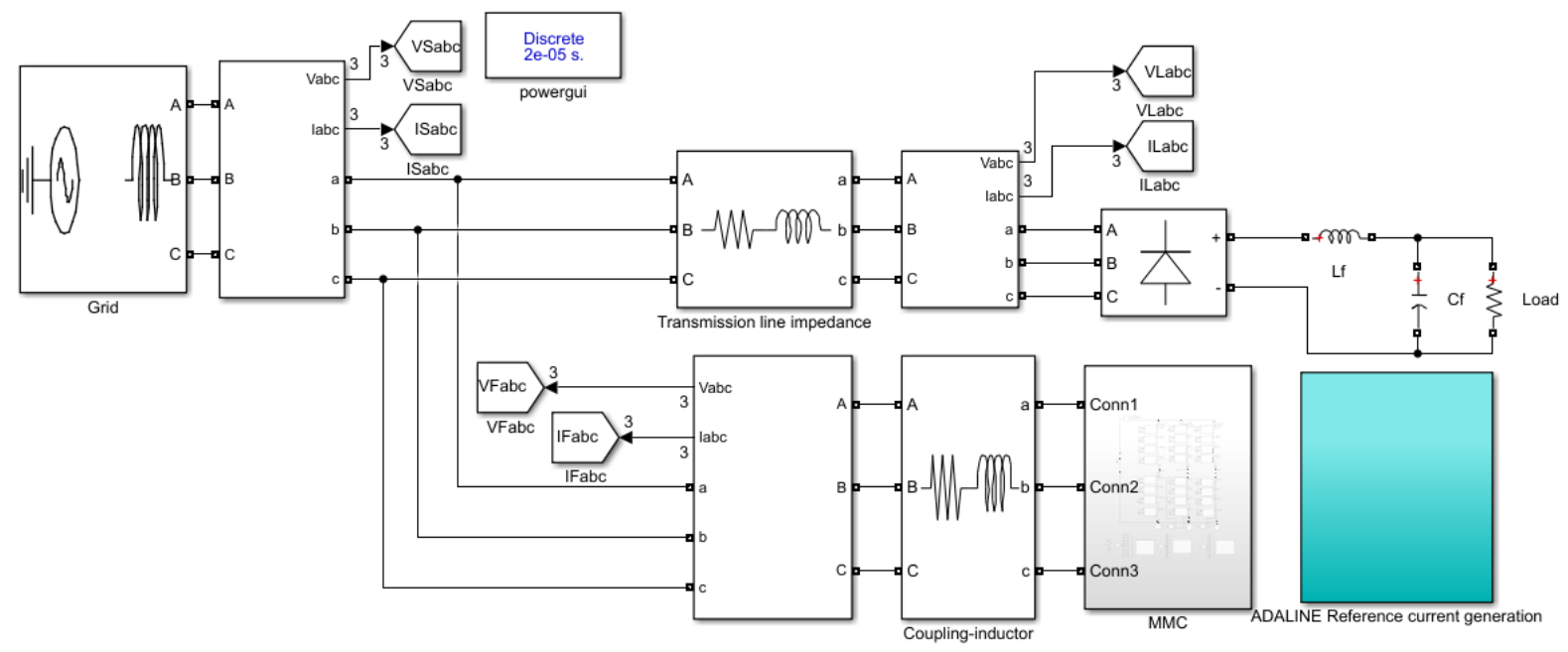

\subsection{SAPF Results}

Figure 9 shows the system Voltage throughout the system while Figure 10 shows the charging of capacitor Submodule around Vdc $/ 4=1250$ volt.

Figure 9: System Voltage

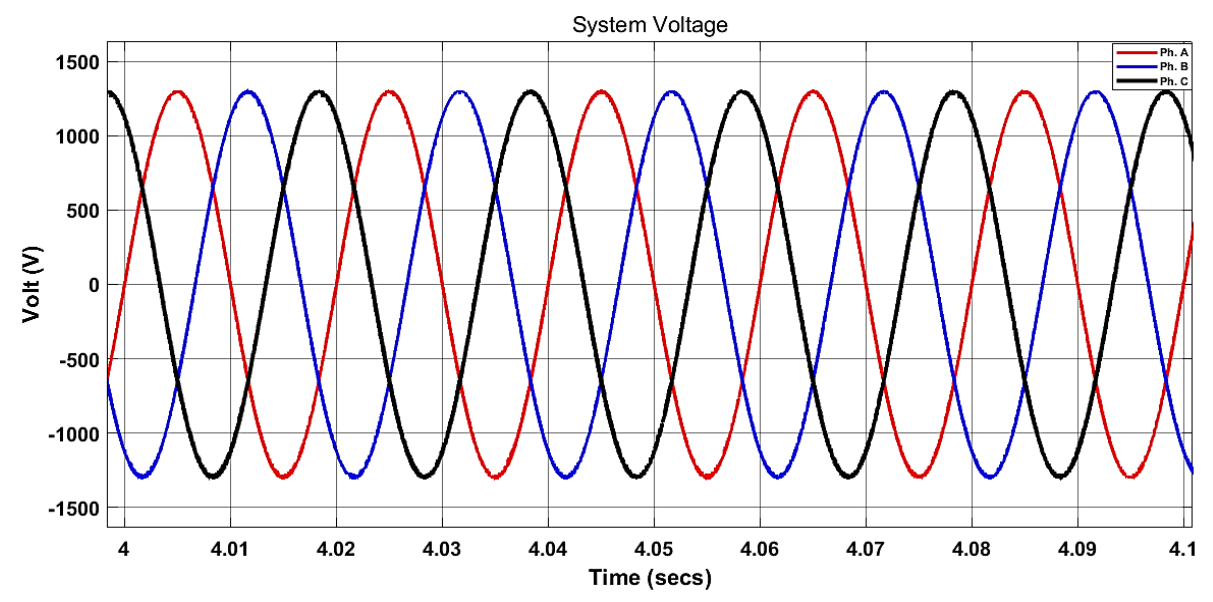

Figure 10: SM capacitor voltage charging and discharging (1290- 1320 V) 


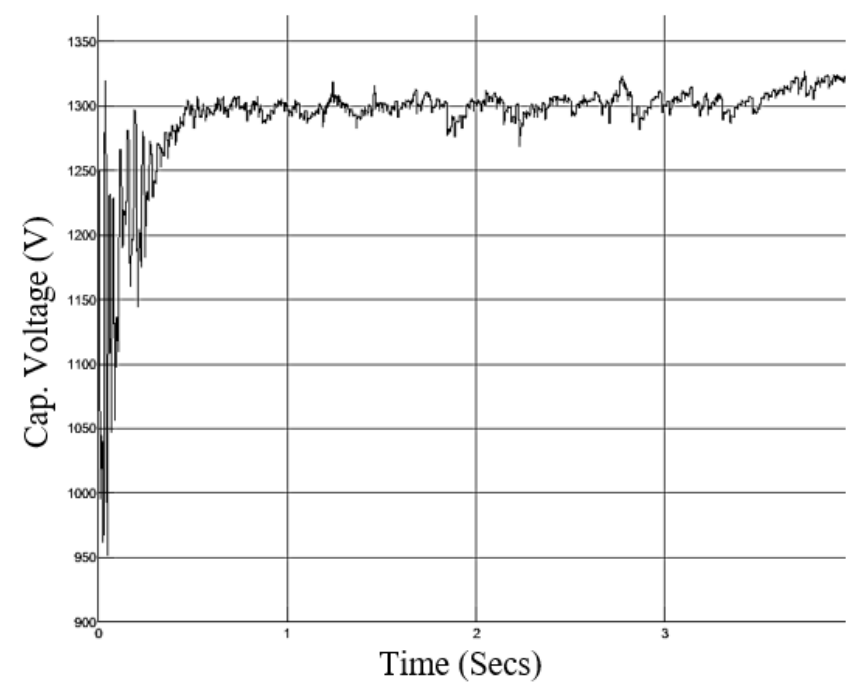

Figure 11 shows the waveform of the source current drawn by the load with no filtering. Owing to the DC nature of this load the source current carries harmonics distorting its waveform. The current THD is 23\%. Applying FFT shows that the load current is a combination of pure sinusoidal wave and high order harmonics, as aforementioned, the MMC active filter should inject the higher-order harmonics enabling the grid to supply only the fundamental wave.

Figure 11: Load Current with no filtering

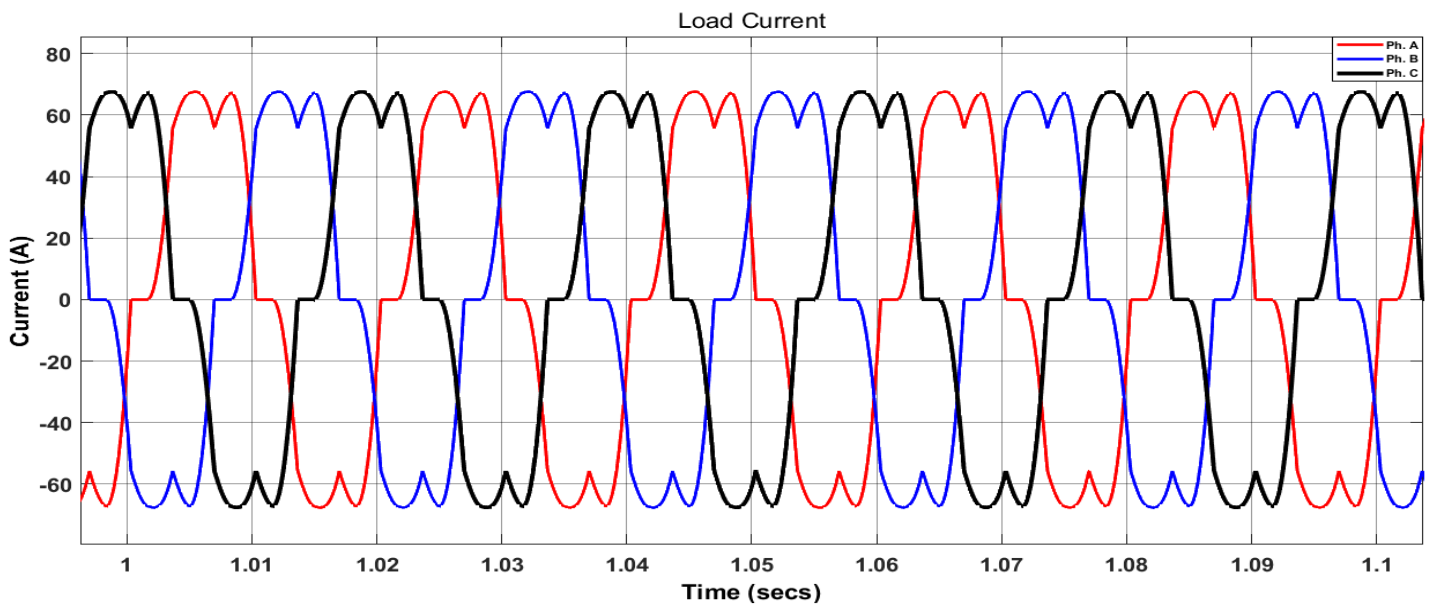

Figure 12 illustrates the current waveforms for the grid, filter, and load currents. Noticeable that the grid supplies a nearly pure sinusoidal wave highlighting the role of the MMC SPAF in mitigating the harmonics to an acceptable value with a THD $\simeq 3 \%$ complying with the IEEE standard 519-2014 (Committee et al., 2014) stipulating that the maximum THD should be below $5 \%$.

Figure 12: Currents of a) Grid current (IS), b) Filter current (If) and C) Load Current after filtering (IL) 

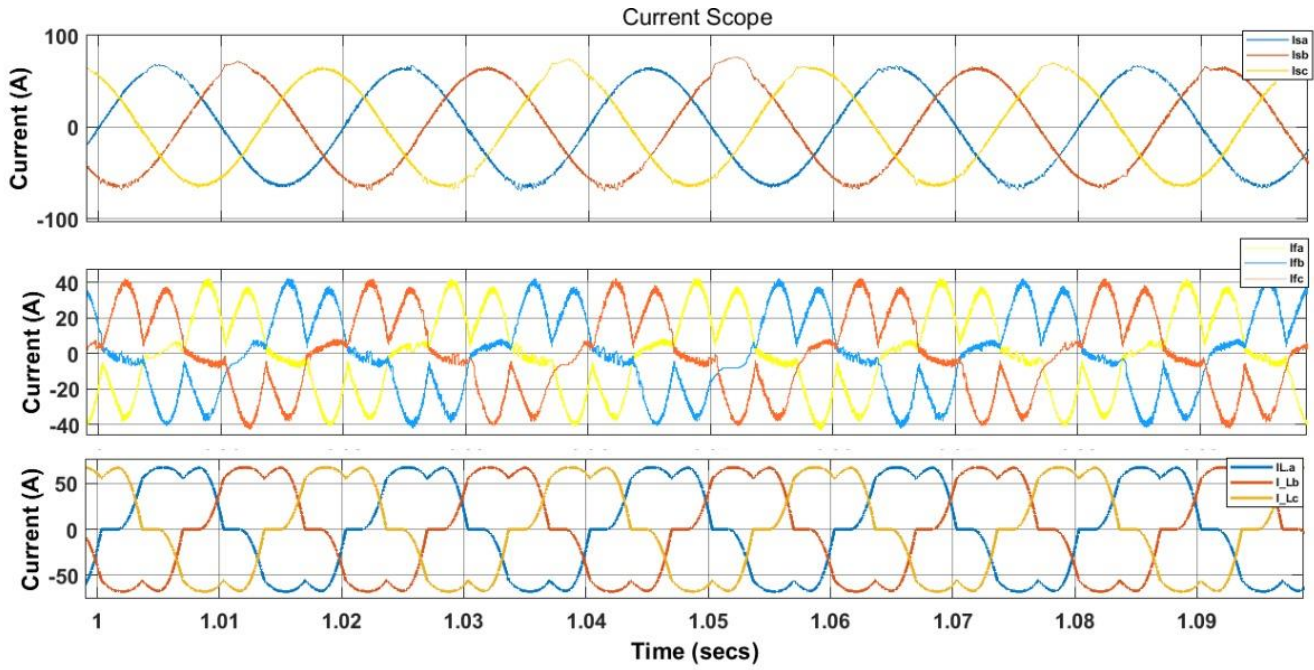

Figure 13: FFT for the Source current

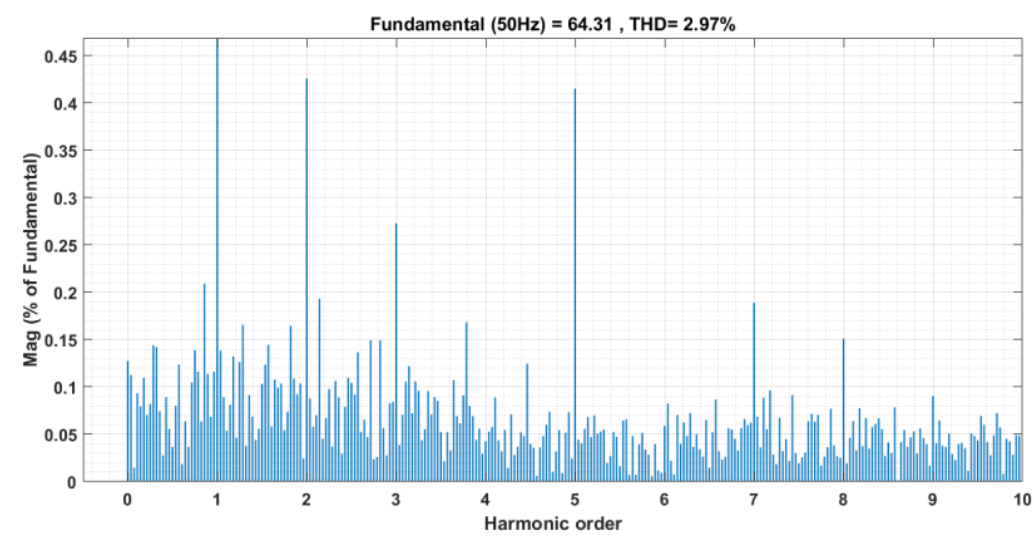

Figure 14: THD-time variance

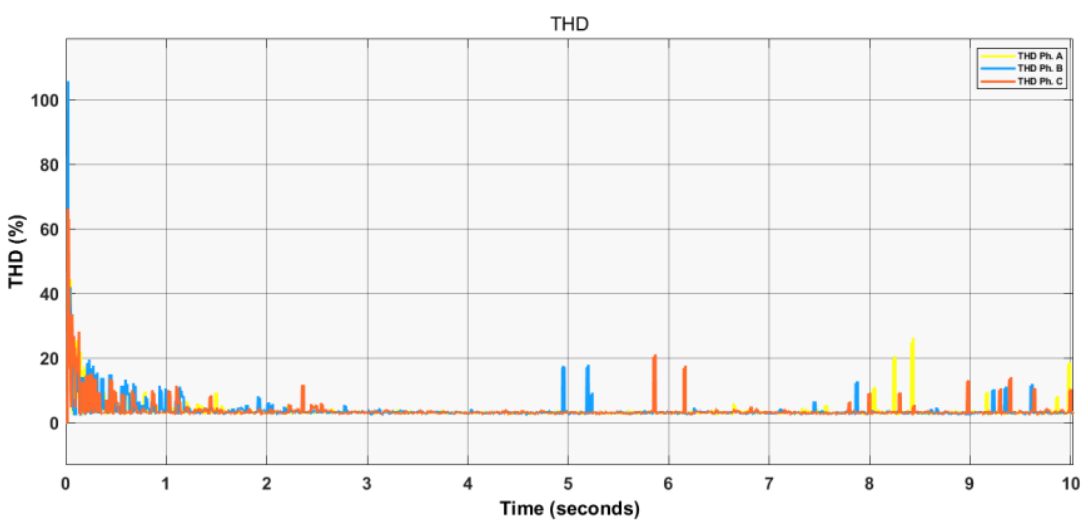

\subsection{Conclusions}

MMC had demolished most disadvantages of the conventional converter, providing additional reliability in various aspects. Using MMC allowed to control, monitor, and report accurately switching frequency. It opened the gate for using algorithmic control for the converter itself or the output signal. In this research, ADALINE is used to extract the reference signal for MMC, controlling switching frequency with different approaches and purifying sensors readiness from noises or ripples. In SAPF application using a double algorithm of ADALINE for a good reference signal without phase shift to be MPC reference. This technique 


\section{$4^{\text {th }}$ International Conference on Modern Research in Science, Engineering and Technology}

5-7 MARCH 2021

BERLIN, GERMANY

maintains a robust reference over wide power applications without being affected, unlike using PI or MPC controllers which proved to be a good controller with a limited power range.

\section{References}

Adam, G. P., Anaya-Lara, O., Burt, G. M., Telford, D., Williams, B. W., \& McDonald, J. R. (2010). Modular multilevel inverter: Pulse width modulation and capacitor balancing technique. IET Power Electronics, 3(5), 702-715.

Cherif, A. Y., Islam Remache, S. El, Barra, K., \& Wira, P. (2019). Adaptive Model Predictive Control for Three Phase Voltage Source Inverter using ADALINE estimator. Proceedings - 2019 IEEE 1st Global Power, Energy and Communication Conference, GPECOM 2019, 164-169.

Chuco, B., \& Watanabe, E. H. (2011). Back-to-back HVDC based on Modular Multilevel Converter. COBEP 2011 - 11th Brazilian Power Electronics Conference, 970-976.

Committee, D., Power, I., \& Society, E. (2014). IEEE Std 519-2014 (Revision of IEEE Std 519-1992), IEEE Recommended Practice and Requirements for Harmonic Control in Electric Power Systems. IEEE Std 519-2014 (Revision of IEEE Std 519-1992), 2014, 1-29.

Coordination, P., Converter, M. M., Design, R., Dc, D., \& Fault, P. (2012). Robust Design During DC Pole to Pole Fault. Ciced, 5-6.

Del Moral, P. H. A. (2020). - Theory and applications. In Mean Field Simulation for Monte Carlo Integration.

Haur, Y. K., Son, T. J., Yun, L. K., \& Raymond, W. J. K. (2020). Design of single-tuned passive harmonic filter to meet ieee-519 standard by means of quality-factor manipulations. International Journal of Advanced Science and Technology, 29(1), 1364-1379.

Hoon, Y., Radzi, M. A. M., Zainuri, M. A. A. M., \& Zawawi, M. A. M. (2019). Shunt active power filter: A review on phase synchronization control techniques. Electronics (Switzerland), 8(7), 120.

Jacob, A., Abraham, B. T., Prakash, N., \& Philip, R. (2014). A Review of Active Power Filters In Power System Applications. International Journal of Advanced Research in Electrical, Electronics and Instrumentation Engineering, 3(6), 10253-10261.

Kalaignan, P. T., \& Raja, S. R. T. (2010). Harmonic elimination by Shunt active filter using PI controller. 2010 IEEE International Conference on Computational Intelligence and Computing Research, ICCIC 2010, 394-395.

Madi, A. I., Hamad, M. S., Hamdy, R. A. R., \& El-Arabawy, I. F. (2018). Hybrid active power filter with modular multilevel converter. 2017 19th International Middle-East Power Systems Conference, MEPCON 2017 - Proceedings, 2018-Febru(December), 1100-1105.

Martinez-Rodrigo, F., Ramirez, D., Rey-Boue, A. B., De Pablo, S., \& Herrero-De Lucas, L. C. (2017). Modular multilevel converters: Control and applications. Energies, 10(11).

Massoud, A. M., Finney, S. J., \& Williams, B. W. (2004). Predictive current control of a shunt active power filter. PESC Record - IEEE Annual Power Electronics Specialists Conference, 5, 35673572 .

Moon, J. W., Gwon, J. S., Park, J. W., Kang, D. W., \& Kim, J. M. (2015). Model Predictive Control with a Reduced Number of Considered States in a Modular Multilevel Converter for HVDC System. IEEE Transactions on Power Delivery, 30(2), 608-617.

Oleschuk, V., Blaabjerg, F., \& Bose, B. K. (2002). Synchronous control of modular multilevel converters. IEEE International Symposium on Industrial Electronics, 4, 1081-1085. 
Ortmeyer, T. H., Chakravarthi, K. R., \& Mahmoud, A. A. (1985). The effects of power system harmonics on power system equipment and loads. IEEE Transactions on Power Apparatus and Systems, PAS-104(9), 2555-2563.

Perez, M. A., Rodriguez, J., Fuentes, E. J., \& Kammerer, F. (2012). Predictive control of ac-ac modular multilevel converters. IEEE Transactions on Industrial Electronics, 59(7), 2832-2839.

Rohner, S., Bernet, S., Hiller, M., \& Sommer, R. (2010). Modulation, losses, and semiconductor requirements of modular multilevel converters. IEEE Transactions on Industrial Electronics, 57(8), 2633-2642.

Tu, Q., Xu, Z., \& Xu, L. (2011). Reduced Switching-frequency modulation and circulating current suppression for modular multilevel converters. IEEE Transactions on Power Delivery, 26(3), 2009-2017.

Vazquez, S., Leon, J. I., Franquelo, L. G., Rodriguez, J., Young, H. A., Marquez, A., \& Zanchetta, P. (2014). Model predictive control: A review of its applications in power electronics. IEEE Industrial Electronics Magazine, 8(1), 16-31.

Widrow, B., \& Winter, R. (1988). Neural Nets for Adaptive Filtering and Adaptive Pattern Recognition. Computer, 21(3), 25-39.

Zygmanowski, M., Grzesik, B., \& Nalepa, R. (2013). Capacitance and inductance selection of the modular multilevel converter. 2013 15th European Conference on Power Electronics and Applications, EPE 2013, September 2013. 